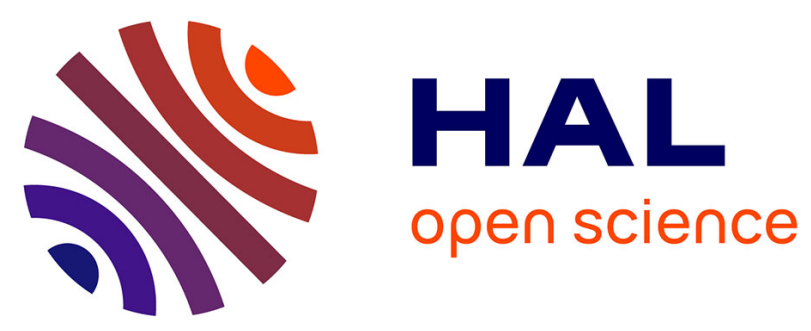

\title{
A Comparison of Several Gradient Based Optimization Algorithms for PAPR Reduction in OFDM Systems
}

Badreddin Koussa, Smail Bachir, Clency Perrine, Claude Duvanaud, Rodolphe Vauzelle

\section{- To cite this version:}

Badreddin Koussa, Smail Bachir, Clency Perrine, Claude Duvanaud, Rodolphe Vauzelle. A Comparison of Several Gradient Based Optimization Algorithms for PAPR Reduction in OFDM Systems. 2nd International Conference on Communications, Computing and Control Applications, Dec 2012, Marseille, France. hal-00783894

\section{HAL Id: hal-00783894 https://hal.science/hal-00783894}

Submitted on 1 Feb 2013

HAL is a multi-disciplinary open access archive for the deposit and dissemination of scientific research documents, whether they are published or not. The documents may come from teaching and research institutions in France or abroad, or from public or private research centers.
L'archive ouverte pluridisciplinaire HAL, est destinée au dépôt et à la diffusion de documents scientifiques de niveau recherche, publiés ou non, émanant des établissements d'enseignement et de recherche français ou étrangers, des laboratoires publics ou privés. 


\title{
A Comparison of Several Gradient Based Optimization Algorithms for PAPR Reduction in OFDM Systems
}

\author{
Badreddin Koussa, Smail Bachir, Clency Perrine, Claude Duvanaud, Rodolphe Vauzelle \\ University of Poitiers, XLIM-SIC Laboratory, UMR CNRS 7252 \\ URL : http://www.sic.sp2mi.univ-poitiers.fr/ \\ Email: badreddin.koussa@univ-poitiers.fr
}

\begin{abstract}
The aim of this paper is to evaluate and compare different optimization algorithms for Peak to Average Power Ratio (PAPR) reduction in Orthogonal Frequency Division Multiplexing (OFDM) systems. Based on Tone Reservation (TR) method, we exploit the unused subcarriers of the studied standard to generate the peak canceling signal without data rate loss. Gradient, Conjugate-Gradient with two directions search and QuasiNewton methods have been investigated and evaluated on the basis of spectral regrowth, convergence speed and ability to improve the high peak-to-average reduction in multicarriers systems. As an example, the simulations are performed in the case of Local Area Network WLAN (IEEE 802.11a standard). Simulation results show that a PAPR reduction gain around $3 \mathrm{~dB}$ can be achieved.
\end{abstract}

Index Terms-OFDM system, PAPR reduction, Tone Reservation, Gradient based optimization methods.

\section{INTRODUCTION}

OFDM for (Orthogonal Frequency Division Multiplexing) modulation is an attractive technique to mitigate interference problems and delay spread due to frequency selective channels [1]. It offers a high spectral efficiency for data transmission. Accordingly, it has been widely adopted for many telecommunication standards such as DVB, WIMAX, LTE and IEEE 802.11a/g WLAN. Unfortunately, OFDM signals present a high fluctuations due to the destructive and constructive sum of many orthogonal subcarriers in time domain. These temporal fluctuations of transmitted signal can be described by a high Peak to Average Power Ratio (PAPR) value which is currently the most used term. A high PAPR value affects performance of non nonlinear devices [2], especially the High Power Amplifier (HPA), introducing Intersymbol Interference (ISI) caused by the In-Band and Out-OfBand distortions. The overall quality of transmission system is then degraded, which leads to poor Bit Error Rate (BER) performance.

High PAPR value problem could be reduced by operating in the linear region of the HPA transfer characteristic by allowing a large enough amplifier back-off. However, this solution is not efficient in terms of HPA power consumption. In fact, the high efficiency region of HPA corresponds to its saturation zone, where nonlinear effects are the most severe[3]. Another mostly used solution to improve the power efficiency and avoid nonlinear distorsion, is to reduce the PAPR of the signal. Based on this principle, numerous solutions have been proposed in the literature. These methods include Clipping [4][5], Clipping with filtering [6][7], Coding [8], Partial Transmit Sequence (PTS) [9][10] and Select Mapping (SLM) [11][12].

Based on the principle of adding signal technique, [13] proposed another method called Tone Reservation (TR). Originally, the main idea is to reserve a subset of subcarriers called Peak Reduction Tones (PRT), to generate a time domain signal which cancels the peak and minimizes the PAPR. This subset of subcarriers does not carry any information data. Moreover, transmitter and receiver must agree on the number and the position of the reserved subcarriers before transmission by sending Side Information (SI), which decreases the useful Bit rate. To deal with this problem, [14] proposed the use of unused subcarriers defined in the DVB-T standards. Furthermore, this work showed the trade-off between the number of dedicated subcarriers for TR method, the PAPR reduction gain and the spectral efficiency .

The generation of the appropriate time signal for PAPR reduction by TR method has been formulated as a convex optimization problem [13]. To solve this problem, the classical Gradient algorithm has been used in [13][15]. However, this algorithm gives a good performance to the detriment of a slow convergence speed. In parameter identification context, recent researchs [16][17] have shown the importance of performing minimization methods to improve the convergence speed and increase the solution accuracy. In this paper, we investigate the performance of the following gradient-based methods: classical Gradient [13] , 
Fletcher-Reeves Conjugate-Gradient [18], Polak-Ribiére Conjugate-Gradient [19] and the Quasi-Newton method especially Marquardt's algorithm [20]. These four optimization solvers are studied and compared on the basis of spectral regrowth, ability to reduce PAPR, and convergence speed. As an example, we report the results in the case of Wireless Local Area Network WLAN IEEE 802.11a standard where we exploit the 12 unused subcarriers to generate the peak canceling signal, thus avoiding the useful data rate loss.

The remainder of this paper is organized as follow: section II gives a brief overview of OFDM system and PAPR definition. In section III, we review the principle of TR method, while section IV explains the studied optimization algorithms, their formulas and characteristics. Simulation results and comparison are provided in section V. Finally, Section VI concludes the paper and gives some prospects.

\section{OVERVIEW OF OFDM SYSTEM}

An OFDM signal is described as the sum of many independent orthogonal subcarriers which have the same frequency bandwidth $\Delta f$. If we note the data symbols $X_{k}, k=0,1, \cdots, N-1$ as a vector $X=$ $\left[X_{0}, X_{1}, \ldots, X_{N-1}\right]$, where $N$ is the number of subcarriers. The representation of the OFDM signal in the baseband is given by [1]

$$
x(t)=\frac{1}{\sqrt{N}} \sum_{k=0}^{N-1} X_{k} e^{j 2 \pi k \Delta f t}, \quad 0<t \leq T
$$

where $X_{k}$ is the data symbol carried by the $k^{\text {th }}$ subcarrier, and $T$ is the OFDM symbol duration.

At the transmitter, the data signal $x(t)$ is generated by the Inverse Fast Fourier Transform (IFFT) of the vector $X$. Then, the generalization of equation (1) for one OFDM symbol can be written as

$$
x=Q \cdot X
$$

where $Q$ is the IFFT matrix of size $N$.

It is necessary to oversample the OFDM signal by a factor of at least 4 [21] to enable accurate peak detection and give a good time domain representation. According to the central-limit theorem, the OFDM time domain signal follows a Gaussian distribution, which explains the presence of some high peaks in the signal [1]. To evaluate these peaks, the PAPR of the OFDM signal defined below is used

$$
\operatorname{PAPR}(x)_{d B}=10 \cdot \log _{10}\left(\frac{\max \quad 0<t \leq T|x(t)|^{2}}{E\left(|x(t)|^{2}\right)}\right)
$$

where $E(\cdot)$ is the mathematical expectation and $E\left(|x(t)|^{2}\right)$ represents the average power of the signal. We can notice from equation (1) that the PAPR increases with the number of subcarriers.

\section{TONE RESERVATION TECHNIQUE}

The main idea of Tone Reservation technique is to add a time domain signal $c$ to the original signal $x$ to reduce its peaks as shown in figure 1 . The resulting $\operatorname{PAPR}(x+c)$ will be lower than the original $\operatorname{PAPR}(x)$.

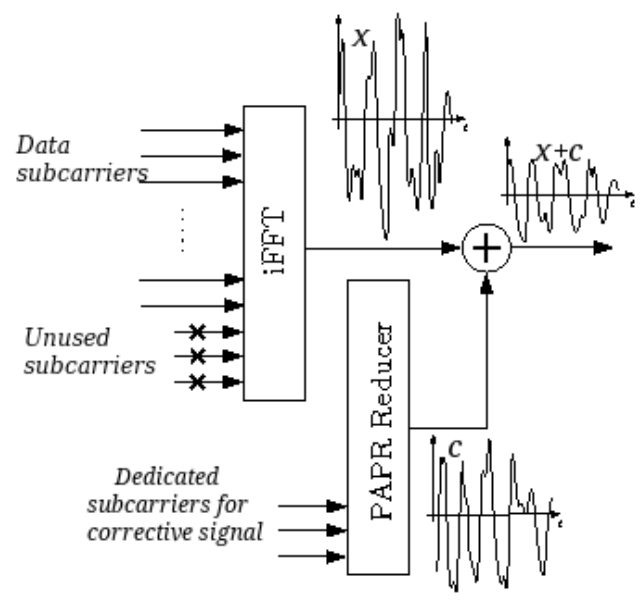

Fig. 1. TR technique using unused subcarriers

From equation (2) we can write [13]

$$
x+c=Q \cdot(X+C)
$$

where $C$ is the verctor of corrective symbols. Therefore, a set of subcarriers is reserved for corrective signal $c$. These subcarriers are dedicated only for peak reduction and do not carry information data. In practical OFDM systems, not all subcarriers are used to transmit useful data, in that case, they can be used for PAPR reduction.

To get the TR as a downward compatible method [22], i.e. reliable without additional information between the transmitter and the receiver, $X_{k}$ and $C_{k}$ must be carried in disjoint frequency subcarriers. Then, we can write

$$
X_{k}+C_{k}= \begin{cases}X_{k} & \text { if } k \in R^{\text {DATA }} \\ C_{k} & \text { if } k \in R^{\text {PRT }}\end{cases}
$$

where $R^{\text {DATA }}$ represents the subset of data bearing and $R^{\text {PRT }}$ represents the subset of subcariers used for PAPR reduction such as $R^{\mathrm{DATA}} \cap R^{\mathrm{PRT}}=\emptyset$

To reduce the PAPR of $x+c$ we must optimize the time domain vector $c$ that minimizes the maximum peak value.

\section{OPTIMIZATION ALGORITHMS}

In [13], authors propose the TR method based on signal to clipping noise power ratio to reduce the complexity of the minimization procedure. According to this principle, we can define the cost function $J$ to be minimized such as

$$
J=\frac{1}{2} \sum_{\left|x_{i}+c_{i}\right|>A} \varepsilon_{i}^{2}=\frac{1}{2} \sum_{\left|x_{i}+c_{i}\right|>A}\left(\left|x_{i}+c_{i}\right|-A\right)^{2}
$$


where $\varepsilon_{i}=\left|x_{i}+c_{i}\right|-A$ is the error between corrected signal $x+c$ and the predefined threshold $A$.

In this cost function, called also quadratic criterion, we consider only samples $i$ exceeding the targeted $A$.

In this context, the optimization problem is to find an optimum value of corrective signal $c$ that minimizes the criterion $J$. In this paper, we evaluate and compare the performance of four iterative optimization algorithms, namely, the Gradient, Conjugate-Gradient with two different directions search and Quasi-Newton methods.

\section{A. Gradient method}

The Gradient algorithm, also called steepest descent method [23][24], is a minimization technique based on a line search in the negative direction of the first derivative of the cost function $J$, also called the gradient. Let $c^{k}$ be the corrective vector at $k^{\text {th }}$ iteration, we proceed to the next correction step of $c^{k+1}$ to minimize the criterion $J$ according to

$$
c^{k+1}=c^{k}-\mu \cdot J_{k}^{\prime}
$$

where $J_{k}^{\prime}=\left[\frac{\partial J}{\partial c}\right]_{c=c^{k}}$ is the value of gradient at the point $c=c^{k}$ and $\mu$ is the monitoring coefficient.

From relation (6), we can deduce by analytical derivation the expression of the gradient such as [13]

$$
J_{k}^{\prime}=\sum_{\left|x_{i}+c_{i}^{k}\right|>A} e^{j \cdot \arg \left(x_{i}+c_{i}^{k}\right)} \varepsilon_{i}^{k} Q q_{i}^{\text {row }}
$$

and the iterative algorithm to update $c$ become

$$
c^{k+1}=c^{k}-\mu \sum_{\left|x_{i}+c_{i}^{k}\right|>A} e^{j \arg \left(x_{i}+c_{i}^{k}\right)} \varepsilon_{i}^{k} Q q_{i}^{\text {row }}
$$

where $e^{j \cdot \arg \left(x_{i}+c_{i}^{k}\right)}$ is the sign function of the complex variable and $\arg (\cdot)$ the angle function. Vector $q_{i}^{\text {row }}$ denotes the $i^{\text {th }}$ row of the iFFT matrix $Q$.

\section{B. Conjugate-Gradient method}

The conjugate gradient method [25][26][27] find an optimal direction search by a combination of the negative gradient at the current iteration and the previous direction. At the $(k+1)^{\text {th }}$ iteration, we update $c^{k}$ according to

$$
c^{k+1}=c^{k}+\lambda^{k} \cdot d^{k}
$$

with $\lambda^{k}$ is conjugate gradient's step, and $d^{k}$ denotes the conjugate gradient direction given as

$$
d^{k}=-J_{k}^{\prime}+\rho^{k} \cdot d^{k-1}
$$

Let us notice that the search direction $d^{k}$ takes into account a previous one $d^{k-1}$ for more efficiency and high convergence speed.

Conjugate gradient methods vary in their computation of the scale parameter $\rho^{k}$, which is used to construct the search direction $d^{k}$. In this paper, two methods are evaluated: The Fletcher-Reeves [18] and Polak-Ribiére methods [24][19] where $\rho^{k}$ can be written as follow

- Fletcher-Reeves method

$$
\rho^{k}=\frac{J_{k}^{\prime} T \cdot J_{k}^{\prime}}{J_{k-1}^{\prime T} \cdot J_{k-1}^{\prime}}
$$

- Polak-Ribiére method

$$
\rho^{k}=\frac{\left({J^{\prime}}_{k}-{J^{\prime}}_{k-1}\right)^{T} \cdot{J^{\prime}}_{k}}{J_{k-1}^{\prime T} \cdot J_{k-1}^{\prime}}
$$

with $(\cdot)^{T}$ denotes transposition function.

\section{Quasi-Newton Method}

Quasi-Newton methods are one of the most effective methods for finding a minimizer of a convex nonlinear function [20][25]. These methods include the curvature along the sequence search directions using the secondderivative information, also called Hessian. The optimal direction search is a vector describing the angle of the direction according to the inverse of the Hessian. Among these methods, Marquardt's algorithm [26][28] was used to ensure an efficient and rapid convergence. In the case of PAPR reduction, the corrective signal $c$ to be estimated are updated as follows

$$
c^{k+1}=c^{k}-\left[J_{k}^{\prime \prime}+\mu \cdot I_{N}\right]^{-1} \cdot J_{k}^{\prime}
$$

$J_{k}^{\prime \prime}=\left[\frac{\partial^{2} J}{\partial c^{2}}\right]_{c=c_{k}}$ is the value of Hessian of criterion $J$. $I_{N}$ represents the identity matrix of size $N$.

In OFDM context, the expression of the Hessian depends on the IFFT matrix $Q$ and the number of used subcarriers such as :

$$
J_{k}^{\prime \prime}=\sum_{\left|x_{i}+c_{i}^{k}\right|>A} Q \cdot Q^{T}
$$

\section{Simulations AND RESUlts}

In this section, we present results from different numerical experiments designed to determine which among the four studied algorithms are preferred according to their convergence speed, PAPR reduction gain and Spectral regrowth.

The simulations are performed using IEEE 802.11a standard. This standard is an extension of the IEEE 802.11 which provides up to $54 \mathrm{Mbps}$ in the $5 \mathrm{GHz}$ band. It uses an OFDM encoding scheme with 64 subcarriers, in which 48 are reserved for information data, 4 reserved for pilots and the remaining 12 are unused [29]. We use these 12 subcarriers to generate the corrective signal $c$. Note that, the proposed algorithms based on TR method can be applied in the case of other standards using OFDM modulation such as IEEE $802.11 \mathrm{~g} / \mathrm{n}$ and LTE, for instance. 


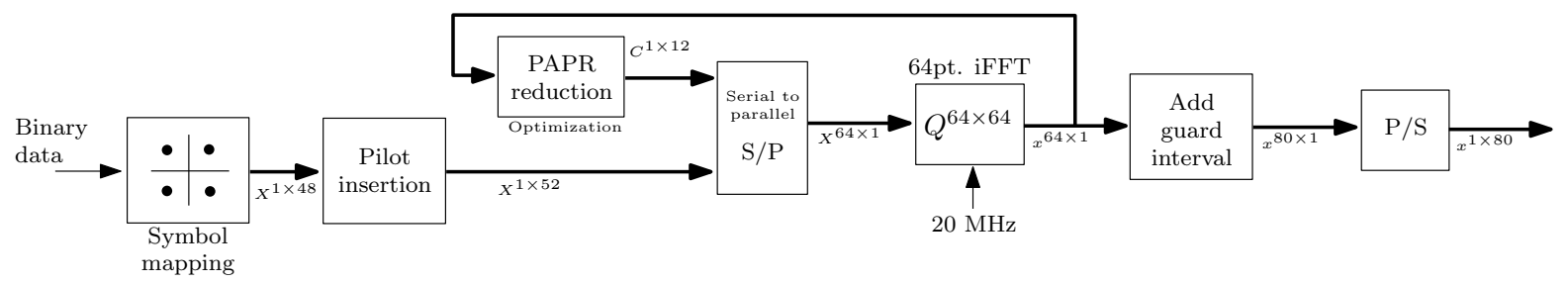

Fig. 2. OFDM data symbol generation and TR scheme for 802.11.a standard

Figure 2 shows the scheme of the simulation, which is carried out for 5000 OFDM symbols modulated by the 16 QAM modulation. The initialization of the corrective signal $c$ is a critical point. Therefore, it is initialized by zero for each OFDM symbol during simulation, allowing a fair comparison of the 4 solvers. Another solution involves updating $c_{i}$ from $c_{i-1}$, where $i$ is the OFDM symbol index's. However, this solution suffers from the problem of algorithm divergence, due to difference of temporal variations from one OFDM symbol to another.

We compare these algorithms through different metrics such as convergence speed, PAPR reduction and spectral regrowth.

Throughout this section, abbreviation Grad denotes the Gradient algorithm (IV-A), Conj-Grad ${ }^{1}$ and Conj$\mathrm{Grad}^{2}$ denote the Conjugate-Gradient with respectively Fletcher-Reeves and Polak-Ribiére methods (IV-B). Quasi-Newton algorithm with Marquardt's version is noted Q-Newton (IV-C).

\section{A. Convergence speed}

To give a quantitative measure of the improvement of convergence speed, we use the normalized mean square error (NMSE), as

$\mathrm{NMSE}_{d B}=10 \log _{10}\left(\sum_{\left|x_{i}+c_{i}\right|>A} \frac{\left(\left|x_{i}+c_{i}\right|-A\right)^{2}}{A^{2}}\right)$

where $i$ is the index of the sample exceeding the threshold $A$. The choice of the $A$ value is related to the temporal fluctuations of the OFDM signal (see figure 4), and the characteristic of the HPA to be used. Therefore, it should be between the maximum of amplitude and the average power of OFDM signal.

To evaluate the convergence speed, the maximum number of iterations is fixed at 50 and the NMSE is computed for the threshold $A=1.1$. Figure 3 shows the NMSE descent during iterations for the 4 studied algorithms.

As shown, all algorithms converge towards the same value and allow an improvement of $7 \mathrm{~dB}$ according to the initial state, except the gradient algorithm, which requires more iterations to achieve the same NMSE

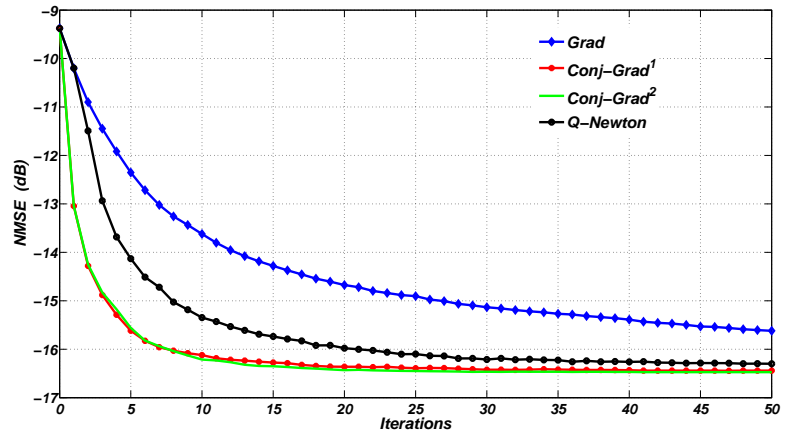

Fig. 3. Comparison of NMSE descent and convergence speed

value. In term of convergence speed, the two versions of conjugate gradient converge and minimize the quadratic error faster than the other algorithms. We can see that minimal value of NMSE is achieved around 10 iterations.

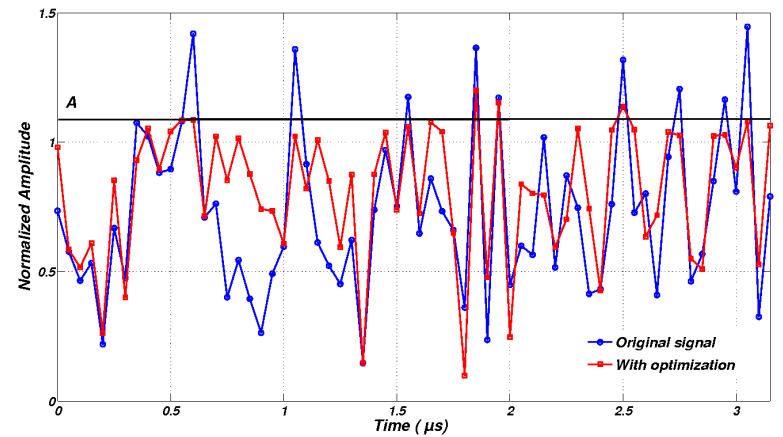

Fig. 4. OFDM time-domain signal with and without optimization

Figure 4 shows the effect of peaks reduction for one OFDM symbol signal in time domain. We can observe that during $3.2 \mu \mathrm{s}$, corresponding to an OFDM Symbol duration specified in the IEEE 802.11a standard before insertion of Guard Interval (GI), the number and the amplitude of peaks have been significantly reduced according to the predefined threshold $A$.

Figure 5 shows the constellation of an OFDM symbol, before and after optimization. We can observe that the peak reducing signal points do not affect the data modu- 
lated symbols, which agrees with the down compatibility principle [22].

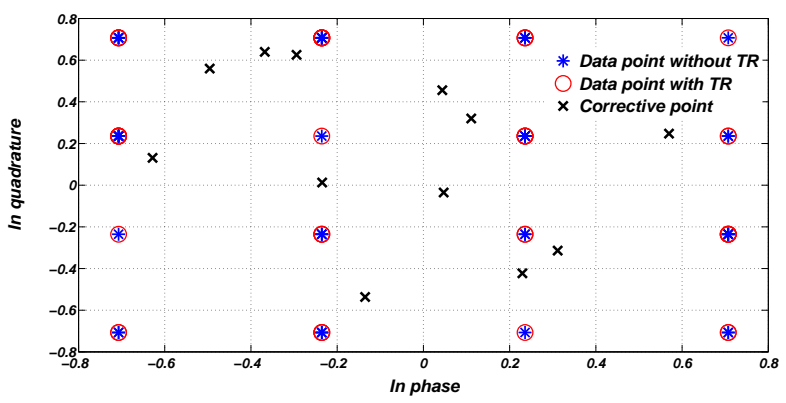

Fig. 5. OFDM symbol constellation for 16QAM modulation

\section{B. PAPR reduction}

Generally, Cumulative Complementary Distribution Function (CCDF) is used to show the variations of PAPR. The CCDF is given by the probability that the PAPR exceeds a given threshold $\mathrm{PAPR}_{0}$ in $\mathrm{dB}$ such as

$$
\operatorname{CCDF}\left(\mathrm{PAPR}_{0}\right)=\operatorname{Pr}\left(\mathrm{PAPR}>\mathrm{PAPR}_{0}\right)
$$

where $\operatorname{Pr}(\cdot)$ denotes probability function.

The following figures show the CCDF of PAPR reduction, the interest is to relate with the descent of NMSE results.

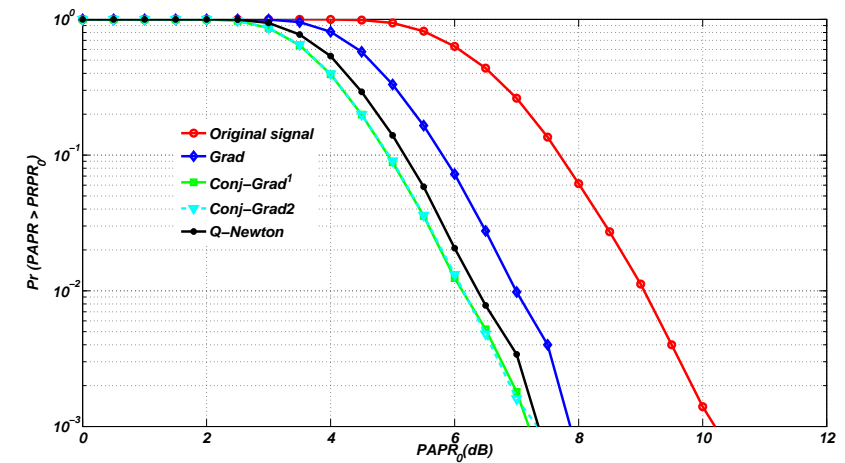

Fig. 6. PAPR reduction comparison of the 4 optimization algorithms after 10 iterations

Figure 6 depicts the CCDF of PAPR reduction after 10 iterations, for the 4 optimization algorithms. It shows that the two versions of conjugate gradient algorithm achieve a good PAPR reduction in fast convergence, compared to Quasi-Newton and gradient algorithms. Figure 7 compares the CCDF of PAPR reduction between the gradient alogrithm and the first version of conjugate gradient algorithm (IV-B) after 5, 10 and 20 iterations respectively. The CCDF curves of conjugate gradient remain nearly unchanged from the $5^{\text {th }}$ iteration, compared to others gradient CCDF curves. Accordingly, the conjugate gradient algorithm reduces PAPR faster than the gradient algorithm. From this figure, we can observe the importance of the convergence speed to get a good PAPR reduction. These results are directly related to the results of the NMSE descent shown in the Figure 3.

Also, as shown in these figures, the TR method with the two versions of conjugate gradient algorithms achieve a PAPR reduction gain around $3 \mathrm{~dB}$ at CCDF of $10^{-3}$. These results are directly related to results of the NMSE descent shown in the Figure 3.

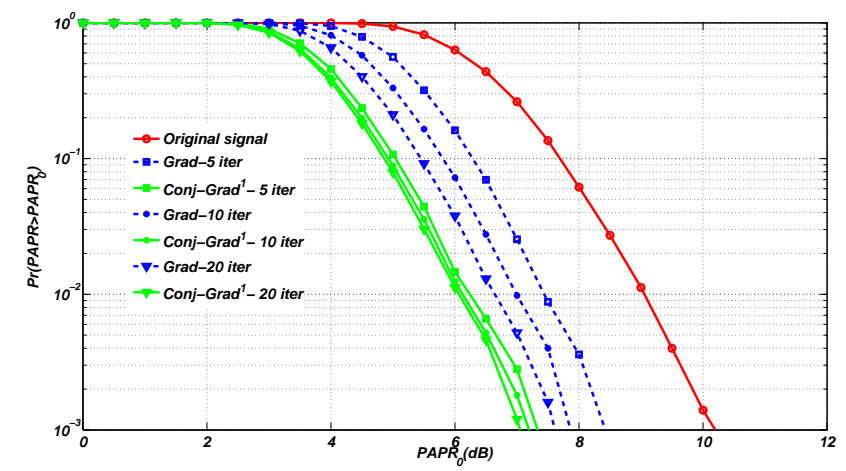

Fig. 7. PAPR reduction comparison of gradient and conjugate gradient algorithms after 5, 10 and 20 iterations

\section{Spectral regrowth}

In practical system, unused subcarriers are reserved to avoid the problem of adjacent channel interference. Generally, these subcarriers are located on the edge of dedicated frequency band. We study the effect of the proposed algorithms for PAPR reduction on the output power spectrum. Figure 8 shows the resulting Power Spectral Density (PSD) for the proposed algorithms.

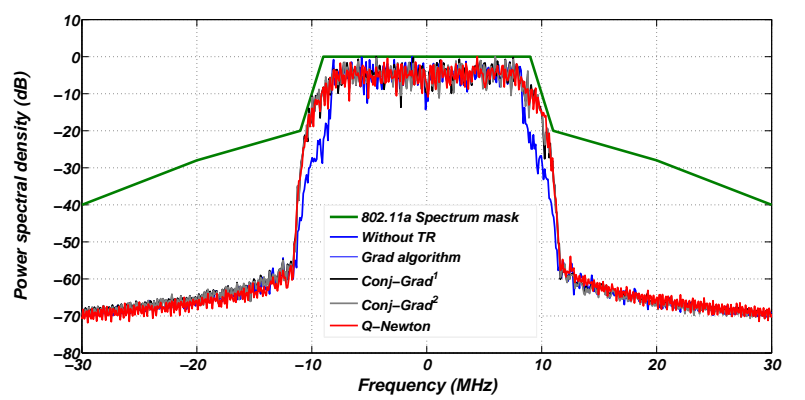

Fig. 8. Power Density Spectrum of OFDM Signals in 802.11a standard

As shown, we can see that TR technique does not affect the PSD distortion, compared to the spectral mask 
defined by IEEE 802.11a standard [29]. Similar results have been obtained, which prove the feasibility of this technique under other frequency specifications [14].

\section{CONCLUSION}

In this paper, the study and the evaluation of four optimization solvers are presented, to minimize the PAPR in the context of OFDM modulation. These proposed solutions are based on the TR method with adding correction in the time domain to the OFDM signal, in order to reduce its peaks. The corrective signal is estimated via these four optimization solvers, namely, Gradient algorithm, Fletcher-Reeves and Polak-Ribiére Conjugate-Gradient and Quasi-Newton methods. Simulation results on the IEEE 802.11a standard show that conjugate gradient solver provides better performance in term of PAPR reduction gain and convergence speed compared to Quasi-Newton and Gradient algorithms. The latter one is commonly used in the literature for TR technique. The power spectrum specifications defined by the standard are respected in all versions of optimization algorithms.

From this study, we can conclude that the investigated optimization algorithms are suitable for PAPR reduction. Our next work is to study and evaluate the impact of these solvers on the HPA power efficiency, which is closely related to green communication development.

\section{REFERENCES}

[1] R. v. Nee and R. Prasad, OFDM for Wireless Multimedia Communications. Norwood, MA, USA: Artech House, Inc., 1st ed., 2000.

[2] S. Bachir and C. Duvanaud, "New identification procedure for continuous-time radio frequency power amplifier model," Journal of Circuits, Systems, and Computers, vol. 19, no. 6, pp. 1259-1274, 2010.

[3] P. B. Kenington, High Linearity RF Amplifier Design. Norwood, MA, USA: Artech House, Inc., 1st ed., 2000.

[4] A. Gatherer and M. Polley, "Controlling clipping probability in dmt transmission," in Signals, Systems amp; Computers, 1997. Conference Record of the Thirty-First Asilomar Conference on, vol. 1, pp. $578-584$ vol.1, nov. 1997.

[5] X. Li and J. Cimini, L.J., "Effects of clipping and filtering on the performance of OFDM," in Vehicular Technology Conference, 1997, IEEE 47th, vol. 3, pp. 1634 -1638 vol.3, may 1997.

[6] Y. Wang and Z. Luo, "Optimized iterative clipping and filtering for PAPR reduction of OFDM signals," Communications, IEEE Transactions on, vol. 59, pp. $33-37$, january 2011.

[7] J. Armstrong, "Peak-to-average power reduction for OFDM by repeated clipping and frequency domain filtering," Electronics Letters, vol. 38, pp. $246-247$, feb 2002.

[8] A. Jones, T. Wilkinson, and S. Barton, "Block coding scheme for reduction of peak to mean envelope power ratio of multicarrier transmission schemes," Electronics Letters, vol. 30, pp. $2098-2099$, dec 1994.

[9] S. Müller and J. Huber, "OFDM with reduced peak-to-average power ratio by optimum combination of partial transmit sequences," Electronics Letters, vol. 33, pp. 368 -369, feb 1997.
[10] N. Taspinar, A. Kalinli, and M. Yildirim, "Partial transmit sequences for PAPR reduction using parallel tabu search algorithm in OFDM systems," Communications Letters, IEEE, vol. 15, pp. 974 -976, september 2011.

[11] R. Bäuml, R. F. H. Fischer, and J. B. Huber, "Reducing the peak-to-average power ratio of multicarrier modulation by selected mapping," Electronics Letters, vol. 32, pp. 2056-2057, 1996.

[12] C.-L. Wang, S.-J. Ku, and C.-J. Yang, "A low-complexity PAPR estimation scheme for OFDM signals and its application to SLM-based PAPR reduction," Selected Topics in Signal Processing, IEEE Journal of, vol. 4, pp. 637 -645, june 2010.

[13] J. Tellado and J. M. Cioffi, "Peak power reduction for multicarrier transmission," in Proc. IEEE GLOBECOM. Conf, 1999.

[14] I. Mahafeno, Y. Louët, and J.-F. Helard, "Peak-to-average power ratio reduction using second order cone programming based tone reservation for terrestrial digital video broadcasting systems," Communications, IET, vol. 3, pp. $1250-1261$, july 2009.

[15] S. Janaaththanan, C. Kasparis, and B. Evans, "A gradient based algorithm for PAPR reduction of OFDM using tone reservation technique," in Vehicular Technology Conference, 2008. VTC Spring 2008. IEEE, pp. 2977 -2980, may 2008.

[16] L. Ljung, System identification: theory for the user. PrenticeHall information and system sciences series, Prentice-Hall, 1987.

[17] S. Choi and D. H. Kim, "Adaptive antenna array utilizing the conjugate gradient method for compensation of multipath fading in a land mobile communication," in Vehicular Technology Conference, 1992, IEEE 42nd, pp. 33 -36 vol.1, may 1992.

[18] R. Fletcher and C. M. Reeves, "Function minimization by conjugate gradients," The Computer Journal, vol. 7, no. 2, pp. 149-154, 1964.

[19] E. Polak and G. Ribière, "Note sur la convergence de méthodes de directions conjuguées," Rev. Fran. Informat. Rech. Opér, vol. 3, no. 1, pp. 35-43, 1969.

[20] R. Schoenberg, "Optimization with the quasi-newton method," Aptech Systems Maple Valley WA, pp. 1-9, 2001.

[21] M. Sharif, M. Gharavi-Alkhansari, and B. Khalaj, "New results on the peak power of OFDM signals based on oversampling," in Communications, 2002. ICC 2002. IEEE International Conference on, vol. 2, pp. 866 - 871 vol.2, 2002.

[22] Y. Louët and J. Palicot, "A classification of methods for efficient power amplification of signals," annals of telecommunications - annales des télécommunications, vol. 63, pp. 351-368, May 2008.

[23] H.-J. Luethi, "Convex optimization: Stephen boyd and lieven vandenberghe," Journal of the American Statistical Association, vol. 100, pp. 1097-1097, 2005.

[24] R. Fletcher, Practical methods of optimization; (2nd ed.). New York, NY, USA: Wiley-Interscience, 1987.

[25] J. Nocedal and S. Wright, Numerical Optimization. Springer Series in Operations Research, Springer, 1999.

[26] D. Luenberger, Linear and nonlinear programming. AddisonWesley, 1984.

[27] M. J. D. Powell, "Some convergence properties of the conjugate gradient method," Mathematical Programming, vol. 11, pp. 42 49, 1976.

[28] D. W. Marquardt, "An algorithm for least-squares estimation of nonlinear parameters," Journal of the Society for Industrial and Applied Mathematics, vol. 11, no. 2, pp. 431-441, 1963.

[29] I. Standards, "IEEE 802.11a." http://standards.ieee.org/findstds/ interps/802.11a-1999.html. 